\title{
Contrast Pattern Mining and Its Application for Building Robust Classifiers
}

\author{
Kotagiri Ramamohanarao \\ Department of Computer Science and Software Engineering \\ The University of Melbourne \\ Kotagiri@unimelb.edu.au
}

\begin{abstract}
The ability to distinguish, differentiate and contrast between different data sets is a key objective in data mining. Such ability can assist domain experts to understand their data and can help in building classification models. This presentation will introduce the techniques for contrasting data sets. It will also focus on some important real world applications that illustrate how contrast patterns can be applied effectively for building robust classifiers.
\end{abstract}

